\title{
THE EFFECTS OF INCREASE AND VARIATIONS IN TIME PRESENTATIONS FOR SECOND LANGUAGE VOCABULARY LEARNING
}

\author{
Radina Mohamad Deli ${ }^{1}$ \\ Zainab Ghareeb-Ali² \\ Shorouk Al-Houti ${ }^{3}$ \\ ${ }^{1}$ Centre for Language Studies, Universiti Malaysia Sarawak \\ ${ }^{2,3}$ Department of Language and Linguistics, University of Essex \\ 1mdradina@cls.unimas.my \\ zghare@essex.ac.uk \\ ${ }^{3}$ skalho@essex.ac.uk
}

\begin{abstract}
Barcroft (2007) found opportunities for word retrieval to be advantageous during second language vocabulary learning. This study extended such a finding and investigated the effect of increased time in target-word retrieval for learning new vocabulary in the L2, as well as the effect of presentation orders of different time conditions on word retrieval. The data were obtained from 17 native Arab speakers who attempted to learn 24 new English words by viewing 24 word-picture pairs. Each picture and its corresponding word were viewed with different time lags of 0,6 and 12 seconds between them in different presentation orders. The results showed that, although the increased time does not positively affect word retrieval, the overall findings correspond to Barcroft's (2007) view, at least in the case of 6 seconds lag. The results also showed that the production of target words in both the control and retrieval-oriented conditions depend on and vary according to the order of presentation, particularly in the case of 6 seconds lag in which word gain is found to be highest when the lag is presented first and second.
\end{abstract}

Keywords: Second language vocabulary learning, word retrieval, time lags

\section{Introduction}

Researchers have long been interested in memory-related phenomena mainly because they constitute a large part of human cognitive processes and their extended importance in the pedagogical domain. As for the present study, interest in both aspects has prompted the replication and expansion of Barcroft's (2007) research on L2 vocabulary learning. Our earlier observation on L2 learners showed 
that a positive effect occurred when opportunities for word retrieval were made available during vocabulary learning. This led to an investigation of whether increased time to provide $L 2$ learners with such opportunities had a positive effect on their vocabulary learning. A further investigation was also carried out in an attempt to examine the effect of different orders of time presentation on word recall. The aim of this study was to find out whether there were significant differences in the recall of target words with different time conditions and presentation order of these in L2 vocabulary learning. The underlying theory in Barcroft's (2007) study, which is drawn from Slamecka and Graf's (1978) generation effect, emphasises that information is better retained when one actively participates in producing it, rather than passively accepting it from an external source (DeWinstanley \& Bjork, 2004). The generation effect has also been replicated in many studies using a variety of generation conditions, materials and memory tests. These studies suggest that aside from single lexical items, generation conditions also enhance memory for meaningful abbreviations, word compounds, numbers, sentences and pictures (see Mulligan \& Duke, 2002).

Extending findings of the generation effect, Mulligan (2006) conducted a study on recall performance improving over repeated recall attempt - a phenomenon known as hypermnesia. The critical theoretical issue of concern was whether hypermnesia is due to repeated testing per se or increased retrieval time, By referring to Roediger and Thorpe's (1978) study, Mulligan concluded that the number of items recalled increased across multiple recall and single long, demonstrating hypermnesia for both pictures and words condition. In relation to the present study, the allocation of time for word retrieval prior to testing, particularly the increase from the control condition ( 0 seconds) to 6 and 12 seconds, mirrors the increased retrieval time in Roediger and Thorpe's study where they provide more opportunities for self-generation of information, which result in better recall of target items. As for the order of presentation, the present study predicted the likely effect on L2 vocabulary learning along the lines of a theoretical perspective on generation effect which states that the most important limiting condition for such an effect is experimental design (Mulligan \& Duke, 2002, p. 1044). Most generation effect studies, however, focus on recall of known words (Barcroft, 2007). Thus far, there are only a few studies dealing particularly with word retrieval of new words although within different contexts of $L 2$ vocabulary learning such as translationbased, picture-based and nonword learning (see Barcroft, 2007; McNamara \& Healy, 1995; Royer, 1973). Barcroft (2007) extended the findings of the previous studies into the domain of picture-based intentional vocabulary learning. The present study maintains that pictures or images are highly advantageous in promoting word retrieval.

McNamara and Healy's (1995) procedural account of the positive generation effect is particularly vital because it extends beyond episodic memory tasks to the acquisition of multiplication skills and most importantly to foreign word acquisition and retention. In their experiment, participants learned nonwords by association with English nouns via different training condition (i.e. read vs. generate) and it was found that the generate condition produced better results. In fact, some participants in the read conditions produced exceptional results, due to self-generating effect of 
recall. As discussed by Barcroft (2007), following this account, generating target words should promote the development of the cognitive processes involved in the retrieval of word forms, which help facilitate word learning. He further discussed the possibility of a rather similar "semantically-cued" strategising of learning and benefit due to the procedural account, for both recall known words and novel words.

The need for more research to be done on the retrieval of new words is an extension of the importance of memory to word-learning. Additionally, the pedagogical bearing of such a research is warranted in that new methods of vocabulary learning involving self-generation, rather than rote-learning has been claimed, much less proven to produce differing results. According to Rohde and Tiefenthal (as cited in Barcroft 2007), processing novel words is different from processing known words due to the optimum "form-meaning" mapping in the former. Hence, there may be a difference in the way generation effect improves memory for novel words and known words, thus the need to investigate the extent in which they differ from each other (Barcroft, 2007).

There were several differences between the present study and Barcroft's. Firstly, the latter only examined two conditions, namely, a control condition (0 second lag) and a retrieval-oriented condition with 6 seconds lag, whilst the former included another condition which is a retrieval-condition with 12 seconds lag. This was to find out whether a longer time lag of 12 seconds would have a similar positive effect for word retrieval. Nonetheless, the amount of time for which the participants were exposed to each individual target word in all conditions remained constant at 6 seconds. The present study only displayed the picture-pair to the participants alongside each other once for three seconds prior to the conditions instead of twice to find out whether this had an effect on word retrieval. There was also the potential presentation order effect to be considered as groups of learners were presented with different lags sequentially. Moreover, a post-test was only administered immediately after the experiment as the focus was not in assessing retrieval performance over time. Different materials were also used to carry out the present study's experiment. Finally, a different $L 1$ group with a different $L 2$, namely Arab speakers, was chosen due to the fact that they can be considered as true beginners who have limited access and exposure to the target language (i.e. English). For instance, in Barcroft's study, English L1 speakers learnt new words in Spanish as an L2.

In the present study, it was hypothesised that allocating more time to provide for opportunities of word retrieval in L2 vocabulary learning and starting out with a longer lag positively affect production of target words. As such, the predictions are as follows:

1. Arab L2-learners of English should produce more target words (or part of them) with 12 seconds lag allocated for word retrieval in vocabulary learning;

2. Starting out with a 12 seconds lag, followed by 6 seconds and no lag allocated for word retrieval in L2 vocabulary learning increases learners' production of target words. 
If there is a significant effect of conditions or different time lags, there are six sets of predictions to be considered in terms of production of target words (i.e. learners' scores), as represented in Table 1.

Table 1

Prediction based on the production of words (scores) in each condition

\begin{tabular}{|l|l|}
\hline No. & Scores ranked by condition \\
\hline 1 & $12 s>6 s>0 s$ \\
2 & $12 s>0 s>6 s$ \\
3 & $6 s>12 s>0 s$ \\
4 & $6 s>0 s>12 s$ \\
5 & $0 s>6 s>12 s$ \\
6 & $0 s>12 s>6 s$ \\
\hline
\end{tabular}

If there is a significant effect of presentation order, there are six sets of predictions to be considered, as shown in Table 2 .

Table 2

Prediction based on the production of words (scores) in each group by order of presentation

\begin{tabular}{|l|l|}
\hline No. & Scores ranked by group (order of presentation \\
\hline 1 & $12 s-0 s-6 s>6 s-12 s-0 s>0 s-6 s-12 s$ \\
2 & $12 s-0 s-6 s>0 s-6 s-12 s>6 s-12 s-0 s$ \\
3 & $6 s-12 s-0 s>12 s-0 s-6 s>0 s-6 s-12 s$ \\
4 & $6 s-12 s-0 s>0 s-6 s-12 s>12 s-0 s-6 s$ \\
5 & $0 s-6 s-12 s>6 s-12 s-0 s>12 s-0 s-6 s$ \\
6 & $0 s-6 s-12 s>12 s-0 s-6 s>6 s-12 s-0 s$ \\
\hline
\end{tabular}

\section{Methodology}

Eighteen native Arab speakers who were learning English as a second language (age $M=26.35$, s.d.= 4.387) participated in this study. All of them were students doing elementary English language courses either at the Colchester English Study Centre or the University of Essex. A majority of the speakers identified themselves as having poor proficiency of English in the questionnaire. Additionally, none of them were able to provide answers for a pretest carried out to assess knowledge of simple English words. They were randomly divided into three groups who did the experiment separately from each other.

The learners were presented with 24 new English words by viewing pictures and their corresponding words, with different conditions; control (C), retrievaloriented (RO) with 6 seconds lag and RO with 12 seconds lag, and different presentation order of these conditions. Most importantly, the processing time for both pictures and words presented together were kept constant in all conditions at 
6 seconds. The pictures used were simple black-and-white drawings based on an existing vocabulary learning material by Snodgrass as well as some which were randomly searched for through Google Images. All 24 words were concrete nouns (see Appendix) that were randomly sorted in terms of domain, unlike those used in Barcroft's study which mostly come under household tools. The words vary in number of syllables (1,2 and 3) and length to reflect words in real life and in each set of eight words, the total number of syllables was set to be approximately similar. Accordingly, the words were also controlled for frequency and difficulty. A pretest containing these words and additional distracters amounting to 30 words were also used. There was also an answer sheet comprising pictures numbered 1 to 24 for the post-test.

Table 3

Conditions and their order of presentation

\begin{tabular}{|l|lll|}
\hline & Conditions & & \\
\cline { 2 - 4 } Order & C & RO & RO \\
Presentation & (0 seconds lag) & (6 seconds lag) & (12 seconds lag) \\
(Group 1/2/3) & RO & RO & C \\
& $(6$ seconds lag) & $(12$ seconds lag) & (0 seconds lag) \\
& RO & C & RO \\
& $(12$ seconds lag) & (0 seconds lag) & (6 seconds lag) \\
\hline
\end{tabular}

A consent form, a language-background questionnaire and written instructions were provided for the participants prior to the experiment, whilst an answer sheet (post test) with numbered pictures and blanks were provided following it. The DMDX software was used to run exact presentation time of pictures and target words on a projected screen. This procedure was simpler and possibly more accurate than the original study's use of flashcards and cassette tapes. There were no practice sessions and breaks during the experiment. Accordingly, the participants were learners with all 24 picture-pair alongside each other only once for three seconds prior to presentation of the conditions instead of twice to control for tiredness effect, which could have simultaneously affected word retrieval.

There were two levels of scoring; syllable score and letter position score. Syllables in each word were scored out of 1 . For example, a score of 0 will be awarded for any incorrect letters to represent monosyllabic words (e.g., "peard" or "kear" for pear). A different syllable scoring was opted for ease of totalling each word with the score of 1 . Furthermore, the present study experimented with a different language than in the original study. The English language does not have a very transparent grapheme-phoneme relationship as opposed to Spanish. However, the present study replicated Barcoft's (2002) scoring protocol for letter position with minor alterations by deducting 1 mark from total letter scores for each additional and unnecessary letter. For example, if "shark" is spelt as "sharkp", 1 mark is deducted from the total letter score $5=4 / 5$.

Two items, namely, item 4 "dustpan" and 5 "trophy", were removed from the data because the total score mean for the two words in both the syllable and 
letter position scoring fell below $15 \%$. Consequently, only the data from 22 out of 24 words were used. Additionally, on the basis of poor performance (below $20 \%$ in both scoring), the data from one participant in group 1, which is the group presented with the 0,6 and 12 seconds lag presentation order, was excluded leaving the group with only five participants which reduced the total number of participants to 17 . The scores were submitted to repeated measures ANOVA by subject and by item. The order of presentation was counterbalanced using a Latin Squares design which resulted in three groups of people experiencing three sets of presentation orders; group 1 with $0 s-6 s-12 s$ lags, group 2 with 6s-12s-0s lags and group 3 with $12 \mathrm{~s}-0 \mathrm{~s}-6 \mathrm{~s}$ lags.

Table 4

\section{Results}

Mean of syllable scoring by condition

\begin{tabular}{|l|c|c|c|c|c|c|}
\hline \multirow{3}{*}{ Group } & \multicolumn{9}{|c|}{ Condition } \\
\cline { 2 - 7 } & \multicolumn{2}{|c|}{ 0s } & \multicolumn{2}{|c|}{6 s } & \multicolumn{2}{c|}{$12 \mathrm{~s}$} \\
\cline { 2 - 7 } & $M$ & sd & $M$ & sd & $M$ & sd \\
\hline $1: 0-6-12$ & .52 & .31 & .57 & .24 & .46 & .26 \\
$2: 6-12-0$ & .30 & .24 & .53 & .34 & .38 & .34 \\
$3: 12-0-6$ & .28 & .22 & .29 & .20 & .44 & .23 \\
Mean Total & .35 & .26 & .42 & .30 & .43 & .27 \\
\hline
\end{tabular}

Table 5

Mean of letter position scoring by condition

\begin{tabular}{|l|c|c|c|c|c|c|}
\hline \multirow{3}{*}{ Group } & \multicolumn{9}{|c|}{ Condition } \\
\cline { 2 - 7 } & \multicolumn{2}{|c|}{ 0s } & \multicolumn{2}{c|}{ 6s } & \multicolumn{2}{c|}{$12 \mathrm{~s}$} \\
\cline { 2 - 7 } & $M$ & sd & $M$ & sd & $M$ & sd \\
\hline $1: 0-6-12$ & .68 & .28 & .68 & .18 & .61 & .22 \\
\hline $2: 6-12-0$ & .52 & .22 & .71 & .17 & .54 & .25 \\
\hline 3: 12-0-6 & .47 & .15 & .31 & .20 & .57 & .19 \\
\hline Mean Total & .54 & .22 & .54 & .25 & .57 & .21 \\
\hline
\end{tabular}

Tables 4 and 5 represent the syllable (S) and letter position (LP) score means respectively based on condition ( $0 \mathrm{~s}, 6 \mathrm{~s}$ and $12 \mathrm{~s}$ lags) in total as well as by group. The repeated measures ANOVA by subject found no significant effect of condition for both S score and LP score (see Appendix). The main effect of group on the scores was also non-significant for S and LP. However, the condition $\times$ group interaction was significant for the LP score and a near significant effect for the $S$ score. All pairwise comparisons were done using parametric tests. Within-subject pairwise comparisons using the paired-sample $t$-test indicated no statistically significant differences between the mean scores of $S$ and LP. Between-subject pairwise 
comparisons using the independent-sample $t$-test, however, revealed a significant difference between group 1 and group 3 in the 6 s lag score means for $S(p=.28)$ and LP $(p=.012)$, and a significant difference between group 2 and group 3 for $6 \mathrm{~s} \mathrm{lag}$ score means only for $L P, p=.004$ and a relatively significant one for $S, p=.085$ ). As for the ANOVA by item, the results revealed no significant effect on condition for both $S$ and LP score. The main effect of group on the scores was also non-significant for $S$ and LP. There was again, however, a significant interaction of condition $\times$ group of in both $S$ and LP scores. Within-subject pairwise comparisons using the paired-sample $t$-test indicated no statistically significant differences between the mean scores of $S$ and LP. Between-subject pairwise comparisons using the independent-sample $t$-test, however, revealed significant differences between group 1 and group 3 in the control (0s lag) score means for $S(p=.015)$ as well as a significant difference in the 6 seconds lag condition for LP ( $p=.008)$ and a near significant one for $S(p=.057)$. There was also a significant effect for $6 \mathrm{~s}$ lag between group 2 and 3 for $S(p=.021)$ and LP $(p=.014)$.

\section{Discussion}

Generally, the result for conditions can be summarised as follows: providing learners with longer time lags to retrieve target words have no effect on their L2 vocabulary learning and the same is true for order of presentation, which means that the overall findings invalidate the earlier hypothesis. The prediction that a longer lag for word retrieval and starting with such a lag (i.e. 12 seconds) will aid the Arab learners to produce more words are thus nullified. These findings seem to oppose results from Barcroft's (2007) study which reflects Slamecka and Graf's (1978) positive outlook on generation effect. However, such a hypothesis should not be accepted at face value on the basis that there was a significant and a near-significant effect of interaction between the conditions and groups of different presentation order. This may indicate that there was a different kind of condition effect occurring when presentation order was taken into consideration and that this cannot be concluded as completely different from Barcroft's (2007) hypothesis. As for the non-significant effect of condition, it could be attributed to the small sample size used in this experiment.

Based on the overall score mean, a trend in which learners perform better with a lag of 12 seconds was observed. However, based on individual group score means, the control condition and the retrieval-oriented conditions were found to produce varied results across conditions. There was an observable trend for when the control condition and the control-oriented conditions were presented in the final position-participants' scores were lowest in these conditions. When the retrieval-oriented conditions were presented first, participants scored highest in these conditions. Conversely, for the control condition, when it was presented in the initial position, participants still performed better with 6 seconds lag but not with 12 seconds lag as it was presented last for the syllable score, but for the letter position score, the control condition produced similar results as the 6 seconds lag. Although the findings pertaining to order of presentation suggest no effect on word production thus rejecting the prediction of 12-6-0 presentation order being the best 
sequence, a consideration must be made for the condition interaction with the presentation order, especially in the case of 6 seconds. As there was a significant difference of score mean for 6 seconds lag when it was presented in the initial and middle when compared to the final position, this confirms that there is a detrimental effect particularly for the 6 seconds lag when it is presented in the final position. However, it should also be taken into account that in group 3 in which the 6 seconds lag was presented last, the participnats may have experienced tiredness or habitual effect. They had gotten used to having a longer lag initially and was disorientated when presented with no lag soon after, that by the time they got to the 6 seconds lag they lost interest in attempting to produce correct answers. In conclusion, the production of target words in L2 vocabulary learning in both the control and retrieval-oriented conditions is affected by the order of presentation, at least for the 6 seconds lag in that when it was designed to be presented last immediately after a 0 seconds lag, learners produced the least target words. As suggested by Mulligan and Duke (2002), experimental design can be a limiting condition for generation effect to take place, which in our case could possibly be the varying order of presentation.

\section{Conclusion}

In general, it can be concluded that increased time to provide for word retrieval does not positively affect learners target word production in L2 vocabulary learning and this is also true in the case of order of presentation in that there were no significant effect found. However, it must be noted that this could have been attributed to the confounding effect produced by the design of the present study which took into account too many conditions as well as presentation orders. However, one particular finding is in line with Barcroft's (2007) claim of a positive effect for opportunities of word retrieval in vocabulary learning as in the case of 6 seconds lag having a significant effect due to presentation order. The fact that 6 seconds lag produced higher word gains in two of the presentation orders except when presented last should not be ignored, although it was only observed as a trend. A good question to ask is whether a longer lag for word retrieval would result in better word gain if the effect of presentation order is eliminated. If so, what is the best way to investigate longer time lags without having to deal with order of presentation effect? Perhaps comparing just two (control and retrieval-oriented) instead of more conditions at one time would be a more viable option for increase in word production.

\section{References}

Barcroft, J. (2002). Semantic and structural elaboration in L2 lexical acquisition. Language Learning, 52(2), 323-363.

Barcroft, J. (2007). Effects of opportunities for word retrieval during second language vocabulary learning. Language Learning, 57(1), 35-56. 
DeWinstanley, P. A., \& Bjork, E. L. (2004). Processing instructions and the generation effect: A test of the multifactor transfer-appropriate processing theory. Memory \& Cognition, 2(6), 945-955.

McNamara, D. S., \& Healy, A.F. (1995). A generation sdvantage for multiplication skill training and nonword vocabulary acquisition. In A. F. Healy \& L. E. Bourne, Jr. (Eds.), Learning and memory of knowledge and skills: Durability and specificity (pp. 132-169). California: Sage Publications.

Mulligan, N. W., \& Duke, M. D. (2002). Positive and negative generation effects, hypermnesia, and total recall time. Memory \& Cognition, 30(7), 1044-1053.

Mulligan, N. W. (2006). Hypermnesia and total retrieval time. Memory, 14(17), 502518.

Roediger, H. L., \& Thorpe, L. A. (1978). The role of recall time in producing hypermnesia. Memory \& Cognition, 6, 296-305.

Royer, J. M. (1973). Memory effects for test-like events during acquisition of foreign language vocabulary. Psychological Reports, 32, 195-198.

Slamecka, N. J., \& Graf, P. (1978). The generation effect: Delineation of a phenomenon. Journal of Experimental Psychology: Human Learning \& Memory, 4, 592-604.

Table 1

\section{Appendix}

Twenty-four word-list used for the study

\begin{tabular}{|l|l|l|l|l|l|}
\hline \multicolumn{2}{|l|}{ Set 1 } & Set 2 & \multicolumn{2}{l|}{ Set 3 } \\
\hline No. & Word & No. & Word & No. & Word \\
\hline 1 & pair & 9 & tie & 17 & grasshopper \\
\hline 2 & beaver & 10 & windmill & 18 & cannon \\
\hline 3 & shark & 11 & thumb & 19 & archer \\
\hline 4 & dustpan & 12 & castle & 20 & cello \\
\hline 5 & trophy & 13 & stove & 21 & comb \\
\hline 6 & bucket & 14 & feather & 22 & rope \\
\hline 7 & hoof & 15 & pyramid & 23 & ladder \\
\hline 8 & rug & 16 & briefcase & 24 & bench \\
\hline
\end{tabular}


Table 2

Statistical value of syllable scores in participant and item analysis

\begin{tabular}{|l|l|l|l|l|l|l|l|l|}
\hline \multirow{2}{*}{ Factor } & \multicolumn{4}{|l|}{ Participant Analysis } & \multicolumn{2}{l|}{ Item Analysis } \\
\cline { 2 - 9 } & F-value & d.f. & p-value & $\begin{array}{l}\text { eta- } \\
\text { squared }\end{array}$ & F-value & d.f. & p-value & $\begin{array}{l}\text { eta- } \\
\text { squared }\end{array}$ \\
\hline FACTOR 1 & 0.732 & 2 & 0.490 & 0.05 & 0.519 & 2 & 0.599 & 0.027 \\
\hline FACTOR 2 & 1.93 & 2 & 0.181 & 0.216 & 1.673 & 2 & 0.214 & 0.15 \\
\hline $\begin{array}{l}\text { FACTOR 1 } \\
\text { X } \\
\text { FACTOR 2 }\end{array}$ & 2.1 & 4 & 0.108 & 0.231 & 4.68 & 4 & 0.005 & 0.33 \\
\hline
\end{tabular}

Table 3

Statistical value of syllable scores in participant and item analysis

\begin{tabular}{|l|l|l|l|l|l|l|l|l|}
\hline \multirow{2}{*}{ Factor } & \multicolumn{9}{|l|}{ Participant Analysis } & \multicolumn{2}{l|}{ Item Analysis } \\
\cline { 2 - 9 } & F-value & d.f. & p-value & $\begin{array}{l}\text { eta- } \\
\text { squared }\end{array}$ & F-value & d.f. & p-value & $\begin{array}{l}\text { eta- } \\
\text { squared }\end{array}$ \\
\hline FACTOR 1 & 0.061 & 2 & 0.941 & 0.04 & 0.131 & 2 & 0.878 & 0.007 \\
\hline FACTOR 2 & 1.09 & 2 & 0.364 & 0.134 & 2.076 & 2 & 0.153 & 0.216 \\
\hline $\begin{array}{l}\text { FACTOR 1 } \\
\text { X } \\
\text { FACTOR 2 }\end{array}$ & 4.65 & 4 & 0.005 & 0.399 & 4.33 & 4 & 0.006 & 0.313 \\
\hline
\end{tabular}

